\title{
Evaluación de la calidad docente y promoción del profesorado (VIII). Legislación universitaria española (f): calidad docente y complementos retributivos (1.ㄹ parte)
}

\author{
Josep Carreras
}

Resumen. Este artículo analiza cómo la legislación universitaria española ha intentado incentivar la función docente estableciendo diversos tipos de retribuciones adicionales para el profesorado. Vigente la Ley de Reforma Universitaria desde el año 1983, en 1989 se modificó el régimen retributivo del profesorado funcionario y se incorporó un componente docente de carácter individual al complemento específico con el fin de incentivar la docencia. Sin embargo, este objetivo no se alcanzó porque la mayoría de las universidades, responsables de evaluar los méritos docentes, aplicaron criterios muy flexibles y generosos, concediendo el complemento docente de forma generalizada y prácticamente automática. La Ley Orgánica de Universidades (LOU), promulgada el año 2001, determinó que el Gobierno y las comunidades autónomas podrían establecer, para el profesorado funcionario y contratado, retribuciones adicionales ligadas a méritos individuales docentes, investigadores y de gestión, y precisó que el Consejo Social de las Universidades, a propuesta de su Consejo de Gobierno, podría acordar la asignación de dichos complementos retributivos, previa evaluación de los méritos por la Agencia Nacional Evaluación de la Calidad y Acreditación o por el órgano de evaluación externa que la comunidad autónoma determinase. Al desarrollar esta disposición, las comunidades autónomas establecieron parámetros y criterios distintos, lo que ha conducido a la coexistencia de situaciones retributivas muy variables. Recientemente, la Ley Orgánica de Modificación de la LOU ha añadido el desarrollo tecnológico y la transferencia de conocimiento a los méritos a que quedaban ligadas las retribuciones adicionales, pero prácticamente no se ha afectado la situación por lo que se refiere a la incentivación de la función docente.

Palabras clave. Complementos retributivos. Incentivación de la docencia. Retribuciones adicionales.

\section{Evaluation of teaching and faculty promotion (VIII). University Spanish legislation (f): quality of teaching} and complementary retributions (1st part)

Summary. This article analyses how the university Spanish legislation has tried to increase the quality of teaching establishing diverse types of additional fees for the professorship. Six years after the promulgation of the Law of University Reformation in 1983, it was modified the remunerative regime of the professorship and an educational component of individual character was incorporated to the fee's specific complement in order to improve the teaching. However, this aim was not achieved because most of the Universities, responsible for evaluating the educational merits of the professorship, applied very flexible and generous criteria; granting the educational complement in a widespread and practically automatic form. The Organic Law of Universities (LOU), enacted in 2001, determined that the Government and the Autonomous Spanish Communities might establish for the academic staff additional retributions linked to teaching, research and management individual merits. And the law also prescribed that the Social Councils of the Universities, at the proposal of their Governing Councils, might agree the assignment of the above mentioned remunerative complements, previous evaluation of the merits by the National Agency for Quality Evaluation and Accreditation or by the external evaluation body that the Autonomous Communities shall determine. In developing this disposition, the Autonomous Communities set different parameters and criteria, which has led to the coexistence of highly variable remunerative situations. Recently, the Organic Law of Modification of the LOU has added the technology development and the transfer of knowledge to the merits linked to additional fee, but has barely affected the situation for what it does to the encouragement of the teaching function.

Key words. Additional fee. Retributive complements. Teaching incentives.
Departamento de Ciencias Fisiológicas I. Facultad de Medicina. Universitat de Barcelona. Barcelona, España.

Correspondencia: Dr. Josep Carreras Barnés. Departamento de Ciencias Fisiológicas I. Facultad de Medicina. Universitat de Barcelona. Casanova, 143. E-08036 Barcelona.

Fax:

+34934035882.

E-mail:

jcarreras@ub.edu

(c) 2011 Educación Médica 


\section{Introducción}

Finalizado el análisis de la legislación relativa a los procedimientos de selección del profesorado universitario, en este artículo comentaremos la normativa sobre los complementos retributivos -de ámbito estatal y autonómico- en cuanto a través de los cuales se ha intentado incentivar la calidad de la actividad docente. Analizaremos, sucesivamente, la normativa derivada de la Ley Orgánica de Reforma Universitaria (LRU) [1], comentada ya en un artículo anterior de esta serie [2], de la Ley Orgánica de Universidades (LOU) [3] y de la Ley Orgánica de Modificación de la LOU (LOMLOU) [4]. Ulteriormente, en la segunda parte de este artículo, resumiremos cómo las diversas comunidades autónomas han desarrollado la aplicación de esta normativa y comentaremos algunos de los informes y estudios que han analizado la situación creada y han realizado propuestas de mejora.

\section{Ley Orgánica de Reforma Universitaria}

\section{Incentivación de la función docente mediante el sistema de retribuciones estatales}

La LRU (Art. 46.1) determinó que correspondía al Gobierno establecer el régimen retributivo del profesorado universitario, el cual tendría carácter uniforme en todas las universidades. $\mathrm{Al}$ año de la promulgación de esta ley, las Cortes Generales aprobaron la Ley de medidas para la reforma de la Función Pública [5], que estructuró las retribuciones de los funcionarios en base a dos componentes (retribuciones básicas y retribuciones complementarias) y estableció, dentro de las segundas, tres complementos: un complemento de destino, correspondiente al nivel del puesto desempeñado; un complemento específico, destinado a retribuir las condiciones particulares de algunos puestos de trabajo, y un complemento de productividad, dirigido a retribuir el especial rendimiento, la actividad extraordinaria y el interés o iniciativa con que el funcionario desempeñara su función. Publicado el año 1985 un Real Decreto que especificó el régimen del profesorado universitario [6], la normativa que fijó las retribuciones de dicho profesorado hasta el año 1988 [7,8] vertebró las retribuciones complementarias de los funcionarios de los cuerpos docentes en régimen de dedicación a tiempo completo sobre la base de la uniformidad de los complementos de destino y específico en razón al cuerpo de pertenencia del profesorado y del desempeño de determinados cargos académicos, sin contemplar entre dichas retribuciones el complemento de productividad. Pero en 1989, con la finalidad de establecer un mecanismo incentivador de la labor docente e investigadora individualizada, se publicó otro Real Decreto [9] que modificó de forma significativa la situación. Por una parte, el complemento específico quedó constituido por tres componentes: un componente general, determinado por el cuerpo docente; un componente singular, determinado por el desempeño de cargos académicos, y un componente por méritos docentes. Por otra parte, se estableció un complemento de productividad basado en la actividad investigadora. La actividad investigadora sería evaluada por una Comisión Nacional integrada por representantes del Ministerio de Educación y Ciencia y de las comunidades autónomas con competencias en materia universitaria -denominada Comisión Nacional Evaluadora de la Actividad Investigadora (CENAI) - y la actividad docente sería evaluada por las universidades de acuerdo con los criterios generales que establecería el Consejo de Universidades. Una evaluación inicial valoraría globalmente los méritos derivados de la actividad realizada hasta el momento, desarrollada en un marco retributivo que no incluía la incentivación, y a partir de la misma tendrían lugar evaluaciones periódicas: los méritos de docencia se evaluarían en períodos de cinco años (quinquenios) y los méritos de investigación en períodos de seis años (sexenios), hasta completar un máximo de cinco evaluaciones favorables (que más tarde se amplió a seis).

Como desarrollo de las disposiciones del Real Decreto, una primera resolución de Consejo de Universidades [10] estableció que la evaluación global inicial de la actividad docente del profesorado sólo podría tener dos calificaciones (favorable o no favorable) y que debía estimarse 'suficiente para una evaluación positiva el correcto cumplimiento de las obligaciones docentes, tales como cumplimiento del régimen horario, asistencia a los alumnos, tutorías, docencia en tercer ciclo'. Una segunda resolución del Consejo [11] fijó los criterios generales que deberían aplicar las universidades para la evaluación de la actividad docente. Estableció que deberían valorarse fundamentalmente las actividades de docencia en primero, segundo y tercer ciclo, examinando especialmente el nivel objetivable del cumplimiento de las obligaciones docentes, la congruencia entre los objetivos docentes y la evaluación efectuada de dichos objetivos, la atención y asistencia a los alumnos, y el cumplimiento de los objetivos didácticos. También deberían ser objeto de evaluación las actividades de extensión universitaria, los cursos de perfeccionamiento, la docencia 
en los títulos propios de las universidades y las tareas directamente relacionadas con la docencia en programas de posgrado $u$ otras actividades docentes institucionales de cada universidad, así como la participación en las actividades de servicio a la comunidad universitaria. La resolución especificó que las universidades deberían establecer los procedimientos de evaluación que estimasen oportunos, guardando la correspondiente coherencia con el proceso evaluador general de su profesorado determinado por la LRU (Art. 45.3), y teniendo en cuenta las condiciones objetivas en las que se realizase la función docente. Indicó que podrían utilizar como instrumentos para la evaluación:

- Una encuesta de los alumnos dirigida a evaluar la actividad estrictamente docente del profesorado, realizada con un modelo suficientemente contrastado y publicado previamente.

- Un informe del propio profesor relativo a su práctica docente, elaborado una vez conocido el resultado de las encuesta de los alumnos.

- Informes de los órganos unipersonales o colegiados de la universidad.

- Informes externos a la universidad.

Señaló, finalmente, que las universidades deberían proveer los mecanismos necesarios para el perfeccionamiento de la docencia, informando a los profesores de las deficiencias observadas para facilitar su corrección con la antelación suficiente a la finalización del período quinquenal de su evaluación.

Pero, como ya comentamos [2], la modificación de los complementos retributivos no produjo los efectos positivos a cuya consecución iba dirigida, especialmente por lo que se refiere a la incentivación de la calidad docente. Para empezar, la normativa promulgada no fue bien recibida, por cuanto se consideró que mezclaba dos problemas que debían resolverse separadamente: la insuficiente retribución del profesorado y la ausencia de procedimientos adecuados para evaluar su rendimiento académico. Por otra parte, se produjo una perversión cuyo riesgo se señaló desde un primer momento: en contraste con la evaluación de la actividad investigadora, realizada por la CENAI con criterios exigentes (aunque criticados por ser poco transparentes y desiguales entre las áreas de conocimiento), la evaluación de la actividad docente realizada por las universidades se hizo con criterios muy flexibles y generosos. En consecuencia, salvo raras excepciones, las universidades reconocieron la bondad de la docencia realizada prácticamente por todo el profesorado, con lo cual se desvirtuó el propósito inicial de la evaluación docente, que se convirtió en un mero instrumento formal para institucionalizar un nuevo complemento salarial por antigüedad. Lo que podía haber sido un mecanismo de incentivación de la docencia se convirtió, en general, en un factor más de su progresivo deterioro. Incluso se abandonaron muchas de las iniciativas internas que se habían puesto en marcha con el fin de evaluar la calidad docente, al cambiar el carácter de las mismas por convertirse el control de la docencia en un mero instrumento administrativo $[2,12]$.

\section{Asignación de complementos \\ retributivos por las universidades}

La LRU determinó que si bien el régimen retributivo del profesorado universitario establecido por el Gobierno tendría carácter uniforme en todas las universidades (Art. 46.1), los Consejos Sociales, a propuesta de las Juntas de Gobierno, podrían 'acordar con carácter individual la asignación de otros conceptos retributivos, en atención a exigencias docentes e investigadoras o a méritos docentes' (Art. 46.2). El Consejo de Estado, en un dictamen emitido en junio de 1998 en respuesta a una consulta referente a este precepto de la LRU formulada por el Gobierno de Canarias [13], insistió en que el establecimiento del régimen retributivo del profesorado universitario era competencia exclusiva y plena del Estado, por lo que las comunidades autónomas no tenían competencias para establecer conceptos retributivos adicionales o el incremento retributivo de los conceptos ya existentes y regulados por la normativa estatal. El dictamen confirmó que el Consejo Social de las Universidades, con carácter individual y en función de circunstancias concretas, podía asignar otros conceptos retributivos distintos de los previstos en el régimen general de retribuciones del profesorado universitario. Pero aclaró que dichos conceptos retributivos no podían operar como incremento de las cuantías previstas para los componentes por méritos docentes y de investigación de los complementos específicos y de productividad establecidos en el régimen retributivo general. $\mathrm{E}$ insistió en que dichos complementos debían ser de carácter individual y estar justificados por la concurrencia de una peculiaridad en el funcionario o en el puesto ocupado referente a aspectos que no estuviesen ya retribuidos específicamente a través del régimen retributivo general. Por ello, no sería conforme al ordenamiento jurídico que la asignación, supuestamente individual, de los nuevos conceptos retributivos determinara como resultado su percepción por la generalidad o mayoría del personal docente de una misma universidad, como tampoco sería ad- 
misible la asignación de conceptos retributivos de aplicación generalizada a colectivos funcionariales por el hecho de su pertenencia a éstos.

Sin embargo, dado que las retribuciones del profesorado universitario eran bajas en comparación con las de los países de la Unión Europea y con las de otros funcionarios del propio Estado y de las comunidades autónomas, la presión de los profesores a través de sus asociaciones y sindicatos propició la concesión de incrementos retributivos vía complementos de productividad o incentivos para la calidad $\mathrm{u}$ otros medios, haciendo una interpretación amplia del artículo 46.2 de la LRU. A partir del año 1995, al producirse el traspaso de competencias a las comunidades autónomas con carácter general y asumir éstas las competencias de financiación de las universidades de su territorio, se llegó a acuerdos que junto a los Consejos Sociales y las juntas y sindicatos de profesores implicaron a las autoridades autonómicas. Las primeras comunidades autónomas que implantaron un sistema de complementos propios fueron Navarra y Canarias, seguidas del País Vasco, Baleares, Madrid y Castilla-La Mancha. Así, a principios del año 2001, seis de las diecisiete comunidades habían implantado algún procedimiento de complementos propios en el sistema retributivo del profesorado universitario, coexistiendo planes de incentivos de carácter individual (pero generalizados) y no consolidables, establecidos con criterios muy dispares y concedidos, generalmente, con una evaluación previa muy generosa o, incluso, de forma automática [13-15].

\section{Propuesta de incorporar un componente autonómico} a los complementos retributivos estatales

Ya en septiembre del año 1999, la Asamblea General de la Conferencia de Rectores de las Universidades Españolas (CRUE) había publicado una declaración en la que se hacía eco de las reivindicaciones del profesorado universitario en relación con su régimen retributivo y solicitaba de los poderes públicos que estudiaran de manera sistemática y total la situación de las remuneraciones del profesorado y del personal de administración y servicios, y 'propusieran en el contexto de un análisis global del problema, una solución que impidiera agravios comparativos e incrementos parciales o individuales' [16].

Unos meses más tarde, los responsables de las administraciones autonómicas, preocupados por la situación que se había creado, solicitaron a la Comisión de Coordinación y Planificación del Consejo de Universidades que se realizara un análisis de la misma y se elaborara algún tipo de propuesta para, en su caso, elevarla al Gobierno. Y, a tal efecto, en octubre del año 2000 se constituyó un grupo de trabajo sobre retribuciones del profesorado universitario. El informe elaborado por este grupo [14] concluyó que el régimen retributivo de dicho profesorado debía modificarse en dos direcciones: una debía dirigirse a incrementar con carácter general y uniforme para todas las universidades algunos de los conceptos retributivos fijados por el Estado; la otra debía ir en el sentido de reconocer la capacidad o competencia de las comunidades autónomas para establecer un régimen propio de complementos basados en incentivos a la productividad por méritos docentes, de investigación, de gestión u otros tendentes a estimular la calidad de las universidades.

El informe consideró que la nueva Ley de Universidades, que el Gobierno estaba preparando, debía contemplar los aspectos mencionados, pero mientras tanto era de la máxima urgencia acometer la posible modificación del Real Decreto sobre retribuciones del profesorado [9] y, en este sentido, propuso dos modificaciones alternativas. La defendida por la mayoría de los miembros del grupo de trabajo suponía añadir al complemento específico un componente autonómico, que podría ser determinado por cada comunidad autónoma para el profesorado de las universidades de su competencia con la finalidad de incentivar la calidad docente, investigadora y de gestión de la universidad. Las comunidades autónomas, dentro de las previsiones de la legislación general, regularían las cuantías de dicho componente y el procedimiento para su concesión que, en todo caso, incluiría la evaluación externa de la actividad del profesorado. La segunda propuesta suponía la adición de un componente autonómico en los complementos específico y de productividad con el que las comunidades autónomas pudieran incrementar su cuantía, pero de forma que se mantuviera la necesaria uniformidad del carácter del régimen retributivo del profesorado.

\section{Ley Orgánica de Universidades}

Como se comenta en su exposición de motivos, uno de los objetivos de la LOU fue articular los distintos niveles competenciales implicados en el sistema universitario En este sentido, la ley dotó de nuevas competencias a las comunidades autónomas; entre otras, la regulación del régimen jurídico y retributivo del profesorado contratado, la capacidad para establecer retribuciones adicionales para el profesorado y la evaluación de la calidad de las universidades de su ámbito de responsabilidad. 


\section{Modificación del régimen retributivo del profesorado}

La LOU se diferenció de la LRU por establecer que mientras el régimen retributivo del personal docente e investigador universitario (PDI) perteneciente a los cuerpos de funcionarios sería fijado por el Gobierno con carácter uniforme en todas las universidades (Art. 69.1), el régimen retributivo del personal docente e investigador contratado en las universidades públicas sería regulado por las comunidades autónomas (Art. 55.1). Estas disposiciones se hallaban ya en el proyecto de ley que el Gobierno remitió a las Cortes y no sufrieron modificación durante la tramitación parlamentaria. Fueron rechazadas las enmiendas del Grupo Parlamentario Vasco, que consideraba contrario a las normas constitucionales la atribución al Estado de la capacidad de regular de modo absoluto y completo todo el régimen retributivo del PDI [17].

\section{Establecimiento de retribuciones adicionales de ámbito nacional}

La LOU no modificó las acciones establecidas para incentivar la docencia y la investigación mediante los complementos específico y de productividad de las retribuciones complementarias del PDI funcionario, pero determinó que el Gobierno podría establecer para el PDI funcionario retribuciones adicionales ligadas a méritos individuales docentes, investigadores y de gestión (Art. 69.2), así como programas de incentivo docente e investigador que comprendieran al PDI contratado en las universidades públicas (Art. 55.3). Y especificó que los complementos retributivos derivados se asignarían previa valoración de los méritos por la Agencia Nacional de Evaluación de la Calidad y Acreditación (ANECA) (Arts. 69.3 y 55.2). Cabe señalar que el proyecto de ley remitido a las Cortes no contenía referencia alguna a la valoración de los méritos del profesorado como requisito para la asignación de los complementos retributivos. La Ponencia del Congreso de los Diputados, en base a enmiendas del Grupo Parlamentario de Coalición Canaria, estableció la necesidad de una 'previa evaluación de los méritos por un órgano o comisión de evaluación externa', y la Comisión de Educación, Cultura y Deporte del Senado incorporó enmiendas del Grupo Parlamentario Popular, dando lugar al redactado definitivo [17].

\section{Establecimiento de complementos retributivos de ámbito autonómico}

El proyecto de ley remitido a las Cortes establecía que el Consejo Social, a propuesta del Consejo de Gobierno y con cargo a los presupuestos de la universidad, podría acordar de manera singular e individualizada la asignación de complementos retributivos a profesores de los cuerpos docentes universitarios (Art. 69.3) y al PDI contratado (Art. 55.2), en atención a exigencias docentes, investigadoras y de gestión de especial relevancia. El Grupo Parlamentario Vasco criticó la atribución de esta competencia al Consejo Social por considerar que invadía competencias autonómicas e introducía una distorsión inaceptable sobre el papel funcional de dicho órgano. Presentó una enmienda al art. 69, atribuyendo la competencia de establecer y regular los conceptos retributivos complementarios y adicionales del PDI funcionario a las comunidades autónomas, y otra enmienda al art. 55, suprimiendo el apartado $2 .^{\circ}$. No se aceptó ninguna de estas dos enmiendas, pero el Grupo Parlamentario de Coalición Canaria, argumentando que en consonancia con el sistema de financiación de las universidades públicas parecía más lógico que el establecimiento de complementos retributivos fuera establecido por la normativa de las comunidades autónomas, presentó dos enmiendas de modificación de los arts. 69 y 55 del proyecto de ley, que se incorporaron al texto aprobado por el Congreso de los Diputados. Así, el texto final de la LOU determinó que, como el Gobierno, las comunidades autónomas podrían establecer retribuciones adicionales ligadas a méritos individuales docentes, investigadores y de gestión tanto para el PDI perteneciente a los cuerpos de funcionarios (Art. 69.3) como para el PDI contratado en las universidades públicas (Art. 55.2), precisando que dentro de los límites que para este fin fijasen las comunidades autónomas, el Consejo Social de las Universidades, a propuesta de su Consejo de Gobierno, podría acordar la asignación singular e individual de dichos complementos retributivos (Arts. 69.3 y 55.2). Finalmente, recogiendo una enmienda presentada por el Grupo Parlamentario de Coalición Canaria y otra presentada por el Grupo Parlamentario Popular, la versión promulgada de la LOU especificó que los complementos retributivos establecidos por las comunidades autónomas se asignarían previa valoración de los méritos por la ANECA o por el órgano de evaluación externa que la ley de la comunidad autónoma determinase (Arts. 69.4 y 55.4) [17].

Promulgada la LOU, todas las comunidades autónomas con competencias en educación universitaria incluyeron en la normativa propia las disposiciones derivadas de la legislación estatal [18], pero los parámetros y los criterios para determinar los 
complementos retributivos variaron notablemente de una comunidad a otra: mientras en unos casos los complementos sólo se concedían al profesorado funcionario, en otros se extendían al profesorado contratado; mientras en unas comunidades autónomas la concesión era automática, en otras había que solicitarla; en algunos casos los complementos se acumulaban, mientras que en otros se exigían evaluaciones periódicas; en los casos en que se requería evaluación previa de los méritos, los instrumentos de evaluación, los criterios de valoración y la rigidez de su aplicación variaban de forma significativa; la cuantía de la retribución adicional resultante de la concesión de los complementos variaba de forma improcedente entre las comunidades autónomas, etc. En los últimos años, se han publicado diversos estudios e informes que analizan la situación así creada y presentan propuestas de mejora. Sin ánimo de ser exhaustivos, en la segunda parte de este artículo comentaremos los que consideramos más interesantes [12,19-28].

\section{Ley Orgánica de Modificación de la LOU}

La LOMLOU modificó algunos aspectos de las disposiciones que la LOU había establecido sobre el régimen retributivo del PDI:

- Eliminó la referencia al carácter uniforme que debería tener en todas universidades el régimen retributivo del PDI funcionario (Art. 69.1). Esta disposición se mantenía en el proyecto de ley que el Gobierno remitió a las Cortes [29], pero se suprimió durante su tramitación parlamentaria.

- Amplió el abanico de méritos individuales a los que quedaban ligadas las retribuciones adicionales (de ámbito estatal y autonómico) del PDI funcionario y contratado (Arts. 69.2, 69.3 y 55.2). La LOU había establecido como méritos computables los méritos docentes, investigadores y de gestión. La LOMLOU estableció que las retribuciones adicionales quedaban ligadas a los méritos por el ejercicio de funciones relativas a la actividad y dedicación docente, la formación docente, la investigación, el desarrollo tecnológico, la transferencia de conocimiento y la gestión. Durante la discusión parlamentaria, el Grupo Parlamentario Vasco presentó una enmienda, que fue rechazada, en el sentido de que las retribuciones mencionadas también se ligaran a méritos lingüísticos, con la finalidad de que las comunidades autónomas con idiomas cooficiales pudieran disponer de este instrumento retributivo para lograr la normalización lingüística [30].
- En concordancia con lo anterior, la LOMLOU determinó que los programas de incentivos para el PDI contratado que el Gobierno puede establecer, además de las funciones de docencia e investigación (ya consideradas por la LOU), abarcarán las funciones de desarrollo tecnológico y de transferencia de conocimiento (Art. 55.3).

- Finalmente, la LOMLOU modificó parcialmente lo establecido por la LOU sobre la valoración previa de los méritos como requisito para la concesión de las retribuciones adicionales. Como ya se ha mencionado, la LOU (Arts. 55.4 y 69.4) había determinado que los complementos retributivos que el Gobierno y las comunidades autónomas podían establecer para el PDI de los cuerpos de funcionarios se asignarían previa valoración de los méritos por la ANECA o por el órgano de evaluación externa que la ley de la comunidad autónoma determinara. El primer borrador de la LOMLOU presentado por el Gobierno socialista en octubre del año 2005 [31] modificaba la disposición referente al PDI funcionario (Art. 69.4) en el sentido de sustituir como agente evaluador de ámbito estatal la ANECA por la CENAI. La CRUE, en base a que la evaluación para la concesión de complementos retributivos debería abarcar la docencia, la investigación y la gestión, no consideró procedente la modificación propuesta en el borrador [32] y el anteproyecto de LOMLOU presentado por el Gobierno en mayo del año 2006 [33] recuperó el redactado del art. 69.4 de la LOU, de forma que el proyecto de ley remitido a las Cortes [29] mantenía intactas las disposiciones de aquella ley. En la discusión del proyecto de la LOMLOU producida en la Comisión de Educación y Ciencia del Congreso de los Diputados, el Grupo Parlamentario Catalán (Convergència i Unió) presentó dos enmiendas sobre este tema [30]. Una, referente a la evaluación de los méritos del PDI funcionario, proponía recuperar la formulación del primer borrador de la LOMLOU, sustituyendo la ANECA por la CENAI como agente evaluador de ámbito estatal. La otra enmienda, referente a la evaluación de los méritos del PDI contratado, proponía especificar que para los complementos retributivos estatales la ANECA sería el órgano de evaluación externo, mientras que para los complementos autonómicos dicho órgano sería determinado por la ley de la comunidad autónoma. La primera de estas enmiendas se rechazó, pero la segunda, con una modificación técnica, se incorporó al texto que finalmente sería promulgado (Art. 55.4). Así, de forma que consideramos incoherente, la disposi- 
ción relativa a la valoración previa de los méritos como requisito para la concesión de las retribuciones adicionales acabó teniendo una redacción distinta para el PDI funcionario (Art. 69.4) y para el PDI contratado (Art. 55.4).

Resulta pues evidente que el Gobierno socialista no quiso utilizar la LOMLOU para realizar la necesaria modificación de la normativa sobre el régimen retributivo del PDI universitario, quizás por considerar más oportuno tratar este tema en el futuro estatuto del PDI. En el momento de escribir este artículo, dicho estatuto parece hallarse en la fase final de su redacción y su contenido será objeto de comentario en un próximo artículo de esta serie.

\section{Bibliografía}

1. Ley Orgánica 11/1983, de 25 de agosto, de Reforma Universitaria (B.O.E. de 1-IX-1983); p. 24034-42.

2. Carreras J. Evaluación de la calidad docente y promoción del profesorado (IV). Legislación universitaria española (b): de la Ley de Reforma Universitaria (1983) a la Ley Orgánica de Universidades (2002) (1. ${ }^{a}$ parte). Educ Med 2004; 7: 9-23.

3. Ley Orgánica 6/2001, de 21 de diciembre, de Universidades (B.O.E. de 24-XII-2001); p. 49400-25.

4. Ley Orgánica $4 / 2007$, de 12 de abril, por la que se modifica la Ley Orgánica 6/2001, de 21 de diciembre, de Universidades (B.O.E. de 13-IV-2007); p. 16241-60.

5. Ley 30/1984, de 2 de agosto, de medidas para la reforma de la función pública (B.O.E. de 3-VIII-1984); p. 22629-50.

6. Real Decreto 898/1985, de 30 de abril, sobre régimen del profesorado universitario (B.O.E. de 19-VI-1985); p. 18927-30.

7. Real Decreto $989 / 1986$, de 23 de mayo, sobre retribuciones del profesorado universitario (B.O.E. de 24-V-1986); p. 18516-8.

8. Real Decreto $1084 / 1988$, de 2 de septiembre, por el que se modifica y complementa el Real Decreto 989/1986, de 23 de mayo, sobre retribuciones del profesorado universitario (B.O.E. de 27-IX-1988); p. 28216-7

9. Real Decreto $1086 / 1989$, de 28 de agosto, sobre retribuciones del profesorado universitario (B.O.E. de 7-IX-1989); p. 28653-6.

10. Resolución de 26 de septiembre de 1989, del Consejo de Universidades, por la que se establecen los criterios generales de evaluación del profesorado universitario para la evaluación global establecida en la disposición transitoria tercera del Real Decreto 1086/1989, de 28 de agosto (B.O.E. de 5-X-1989); p. 31352.

11. Resolución de 20 de junio de 1990, del Consejo de Universidades, por la que se establecen los criterios generales para la evaluación de la actividad docente del profesorado universitario prevista en el artículo 2 ${ }^{\circ}$.3.c) del Real Decreto 1086/1989, de 28 de agosto, sobre retribuciones del profesorado universitario (B.O.E. de 30-VI-1990); p. 19726.

12. Tejedor FJ, Jornet FM. La evaluación del profesorado universitario en España. Revista Electrónica de Investigación Educativa 2008. URL: http://redie.uabc.mx/NumEsp1/contenidotejedorjornet.html. [25.05.2009].

13. Consejo de Estado. Dictámenes. Número de expediente: 2061/1998 (Gobierno de Canarias). URL: http://www.boe. es/aeboe/consultas/bases_datos_ce/doc.php?coleccion= ce\&id=1998-2061. [04.08.2010].

14. Comisión de Coordinación y Planificación del Consejo de Universidades. Punto n. ${ }^{\circ} 4$ del orden del día. Informe y propuesta del grupo de trabajo sobre retribuciones del profesorado. 27/02/2001. URL: http://www.uv.es/ugt/interes/ legislacionuniversitaria/comretripdi.htm. [04.08.2010].

15. Informe sobre las retribuciones complementarias del profesorado universitario en las universidades españolas (noviembre de 2002). URL: http://campus.usal.es/ agptu/ docs/Retrcompl/2002/0211Informe.html\%20. [04.08.2010].

16. CRUE. Declaración de la Asamblea General de 27/09/1990. URL: http://www.crue.org/export/sites/Crue/crue/docs/ AGdeclaraciones/DeclaracionCRUEretribucionesprof.pdf. [09.08.2010].

17. Bassols M, ed. Publicaciones de las Cortes Generales. Serie I. Trabajos Parlamentarios. Ley Orgánica de Universidades (Vols. I y II). Madrid: Departamento de Publicaciones de las Cortes Generales; 2004.

18. Embid A, Gurrea F, eds. Legislación Universitaria. Normativa General y Autonómica. 11 ed. Cap. VIII - Comunidades Autónomas. Madrid: Tecnos; 2008.

19. San Segundo MJ. Promoción y remuneración del profesorado universitario. Hacienda Pública Española. Revista de Economía Pública 2005; 172: 93-117.

20. Vaquero A. Políticas de incentivos sobre el profesorado universitario. Situación actual y propuestas de mejora. Presupuesto y Gasto Público 2005; 41: 309-32.

21. Rey JM. Incentivos para la actividad investigadora de los profesores de las universidades públicas españolas. Asociación Española de Ecología Terrestre; 2005. URL: http://www.aeet. org/documentos/Informe_incentivos_inv.pdf. [04.08.2010].

22. Zafra I. El sueldo de un profesor universitario puede variar 10.000 euros según la comunidad. El País, 5-VI-2006.

23. FETE-UGT. Informe sobre el complemento autonómico del PDI laboral de las universidades públicas. Gabinete Técnico; 2007. URL: http://www.ujaen.es/huesped/fete-ugt/documentos/ pdi_uja/pdilaboral/comparativa_situac_pdilab_univ_estatales/ 1informe_completo_Universidad.pdf. [04.08.2010].

24. Murillo FJ. La evaluación del profesorado universitario en España. Revista Iberoamericana de Investigación Educativa 2008; $1: 29-45$.

25. Moretón MF. Selección, evaluación y acreditación del profesorado ante el Espacio Europeo de Educación Superior. Revista de Derecho UNED 2009; 5: 319-71.

26. Escudero T, Pino JL, Rodríguez C. Evaluación del profesorado universitario para incentivos individuales: revisión metaevaluativa. Revista de Educación 2010; 351: 513-37.

27. Jornet JM, Perales MJ, González J. Evaluación de las políticas sobre actividad docente del profesorado universitario. Revista Fuentes 2010; 10: 33-51.

28. Souvirón JM. La evaluación de la actividad docente del profesorado universitario: algunas iniciativas de carácter general en curso. Revista Jurídica de Investigación e Innovación Educativa 2010; 1: 11-24.

29. Proyecto de Ley $121 / 000101$ Orgánica por la que se modifica la Ley Orgánica 6/2001, de 21 de diciembre, de Universidades. Boletín Oficial de las Cortes Generales. Congreso de los Diputados. VIII Legislatura. Serie A, n. ${ }^{\circ}$ 101-1 (8-IX-2006); p. 1-16.

30. Proyecto de Ley $121 / 000101$ Orgánica por la que se modifica la Ley Orgánica 6/2001, de 21 de diciembre, de Universidades. Enmiendas e índice de enmiendas al articulado. Boletín Oficial de las Cortes Generales. Congreso de los Diputados. VIII Legislatura. Serie A, n. ${ }^{\circ}$ 101-9 (28-XI-2006); p. 31-185.

31. Ministerio de Educación y Ciencia. Borrador de la Ley Orgánica de modificación de la Ley Orgánica 6/2001, de 21 de diciembre, de Universidades (27-X-2005). URL: http:// www.crue.org/boletines/boletín_N1/Adjuntos\%20de\%20 AG\%2027-10-05/Borrador_Lou.pdf. [05.05.2008].

32. Acuerdo de la Asamblea General de la Conferencia de Rectores de las Universidades Españolas: modificación de la Ley Orgánica de Universidades. Propuestas sobre profesorado (25-I-2006). URL: http://www.crue.org/ boletines/boletín_ N2/adjuntos/profesorado.pdf.[05.05.2008].

33. Ministerio de Educación y Ciencia. Borrador del anteproyecto de Ley Orgánica por la que se modifica la Ley Orgánica 6/2001, de 21 de diciembre, de Universidades (23-V-2006). URL: http://www.crue.org/boletines/boletín_N7/Boletín\% 20N7\%20/adjuntos/Anteproyecto\%20de\%20reforma\% 20 de\%20la\%20LOU(25-05-06).pdf. [05.05.2008]. 